\title{
A prospective feasibility and safety study of laparoscopy-assisted distal gastrectomy for clinical stage I gastric cancer initiated by surgeons with much experience of open gastrectomy and laparoscopic surgery
}

\author{
Takaki Yoshikawa · Haruhiko Cho • Yasushi Rino • Yuji Yamamoto • \\ Masayuki Kimura - Tetsu Fukunaga - Shinichi Hasegawa - Takanobu Yamada • \\ Toru Aoyama $\cdot$ Akira Tsuburaya
}

Received: 26 December 2011/Accepted: 1 April 2012/Published online: 20 April 2012

(c) The International Gastric Cancer Association and The Japanese Gastric Cancer Association 2012

\begin{abstract}
Background The aim of this prospective study was to evaluate the feasibility and safety of laparoscopy-assisted distal gastrectomy (LADG) initiated by surgeons with much experience of open gastrectomy and laparoscopic surgery.

Methods Three surgeons who each had experience with more than 300 cases of open gastrectomy, more than 100 cases of laparoscopic cholecystectomy, more than 5 cases of laparoscopic colectomy, and more than 5 cases of laparoscopic partial gastrectomy were nominated as LADG operators. All three operators received training for LADG with study materials including videotapes, a box simulator, and an animal laboratory, with lectures and assistance from LADG instructors who each had experience of more than 50 LADG operations. Then the nominated LADG operators performed LADG with the instructors, in which their skills were evaluated and certified. Thereafter, they performed
\end{abstract}

T. Yoshikawa $(\bowtie) \cdot$ H. Cho $\cdot$ S. Hasegawa $\cdot$ T. Yamada.

T. Aoyama $\cdot$ A. Tsuburaya

Department of Gastrointestinal Surgery, Kanagawa Cancer Center, 1-1-2 Nakao, Asahi-ku, Yokohama 241-0815, Japan

e-mail: yoshikawat@kcch.jp

Y. Rino

Department of Surgery, Yokohama City University,

Yokohama, Japan

Y. Yamamoto

Department of Surgery, Kanagawa Ashigawa Kami Hospital,

Kanagawa, Japan

M. Kimura · T. Fukunaga

Department of Gastrointestinal Surgery, St Marianna University,

Kawasaki, Japan
LADG without assistance from the instructors. The target of this study was clinical stage I gastric cancer that was resectable by distal gastrectomy. D1 + alpha, D1 + beta, or D2 dissection was performed laparoscopically. Basically reconstruction was done extracorporeally with a Billroth-I gastroduodenostomy. An extramural review board checked the surgical quality of the operations performed by the three surgeons. The primary endpoint was morbidity and mortality.

Results A total of 193 patients were enrolled in this study between August 2004 and July 2009. The median blood loss was $35 \mathrm{ml}$ and the median operation time was $250 \mathrm{~min}$. Conversion to open surgery was seen in 6 patients; 4 due to bleeding and 2 due to advanced disease. Overall morbidity was $1.6 \%$, including grade 2 anastomotic leakage in $0.5 \%$ and grade 2 pancreatic fistula in $0.5 \%$. No mortality was observed. The number of cases required until the LADG operators acted as LADG surgeons without an instructor was 3 for each of the three surgeons. When comparing the data between that in the training period $(n=9)$ and the operators' data $(n=174)$, the median operation time was significantly longer in the training period $(355 \mathrm{~min})$ than in the latter period (247.5 min) $(p=0.015)$. Median blood loss was also greater in the training period $(150 \mathrm{ml})$ than the latter period $(32.5 \mathrm{ml})$, but the difference did not reach statistical significance $(p=0.084)$. During the training period, no patient developed any complications of $\geq$ grade 2 .

Conclusion These results suggested that LADG could be initiated and performed feasibly and safely if surgeons with much experience of open gastrectomy and laparoscopic surgery received adequate training for LADG.

Keywords Laparoscopy - Gastrectomy - Prospective study · Gastric cancer 


\section{Introduction}

Early gastric cancer is a highly curable disease; thus, there is an increasing demand to improve patients' postoperative quality of life. Kitano first reported laparoscopy-assisted distal gastrectomy (LADG) in 1994 [1]. Initially, the feasibility and safety were not satisfactory [2]. However, several studies have recently demonstrated that LADG is feasible, safe, less invasive than open surgery, and yields good quality of life in addition to having cosmetic benefits [3-6]. Moreover, long-term survival has been evaluated in a retrospective analysis of a case series with a large sample size [7]. Recently, Katai et al. [8], in a phase II study, reported an incidence of $1.7 \%$ each for pancreatic fistula and anastomotic leakage . Based on this finding, they initiated a phase III trial for early gastric cancer to show that the survival of LADG was comparable to that of conventional open distal gastrectomy. Moreover, another phase II/III trial for resectable advanced disease has been launched by a group of leading Japanese laparoscopic surgeons. However, these phase III trials have been performed only by experienced, specifically accredited surgeons.

It may be difficult to expand the application of LADG in Japan if the phase III trials are found to have positive results, because most surgeons in this country have no experience of LADG. However, Japanese surgeons in general hospitals have much experience of open gastrectomy for gastric cancer, and they are also experienced in performing laparoscopic cholecystectomy. Some surgeons have experience of laparoscopic partial gastrectomy and may have experience of colectomy with nodal dissection for colon cancer. Many surgeons would have experience with the use of ultrasonic cutting and coagulating systems when dissecting lymph nodes in open or laparoscopic surgery, opening the omentum in staging laparoscopy, or removing the lesser curvature in laparoscopic partial gastrectomy. Furthermore, as laparoscopic surgery is easily recorded by videotape, this enables surgeons to learn the skill more quickly and efficiently. Although LADG is a technically complex procedure, each part consists of a basic technique. If surgeons have enough experience of gastric cancer surgery and laparoscopic surgery, the performance of LADG might be only a small next step. The aim of the present study was to evaluate whether surgeons experienced in the procedures outlined above could initiate and perform LADG safely and feasibly.

\section{Patients and methods}

\section{Eligibility criteria}

The eligibility criteria for this study included: (1) histologically proven gastric adenocarcinoma; (2) T1N0, T1 with nodal metastases limited to the perigastric nodes, or T2N0 clinically determined by endoscopy and abdominal computed tomography (CT) scan, according to the the Japanese Gastric Cancer Association (JGCA) Japanese classification of gastric carcinoma, 2nd English edition [9]; (3) curative surgery that could be performed by distal gastrectomy; (4) no distant metastases, clinically confirmed by physical examination, chest X-ray, and abdominal CT scan; (4) Eastern Cooperative Oncology Group (ECOG) performance status $0-1$; (5) sufficient organ function (according to laboratory data, and physically able to tolerate surgery); (6) forced expiratory volume in $1 \mathrm{~s}$ of $\geq 50 \%$, expected vital capacity of $>70 \%$, and arterial oxygen pressure in room air of $\geq 94$ torr); and (7) written informed consent. Exclusion criteria included: (1) previous history of upper-abdominal surgery; (2) lower-limb varicose veins needing treatment; (3) presence or previous history of deep vein thrombosis; and (4) uncontrolled arrhythmia, or cardiac disease even when controlled with medication.

\section{Surgery}

Five or six ports were used. Lymph node dissection was performed in the laparoscopic field. The omentum was preserved except where resection was necessary for lymph node dissection along the right gastroepiploic artery. A small abdominal incision $(<7 \mathrm{~cm})$ was made for removal of the specimen and reconstruction. Reconstruction was done with Billroth-I gastroduodenostomy, in principle, but Billroth-II or Roux-en Y gastrojejunostomy was applied for small remnant stomachs. All the reconstruction procedures were performed extracorporeally with circular staplers.

The minimum extent of dissection was determined by the JGCA guidelines [10]. Briefly, the details of nodal dissection and indications were: (1) D1 with nodal dissection along the left gastric artery (\#7) and the common hepatic artery (\#8a), termed D1 + alpha, is indicated for mucosal tumors with a diameter of $<1.5 \mathrm{~cm}$ with differentiated histology; (2) D1 + alpha with nodal dissection around the celiac artery (\#9) is indicated for T1N0 tumors that do not fulfill the indications for D1 + alpha, and this procedure is termed D1 + beta; and (3) D2 dissection, which is indicated for $\mathrm{T} 1$ with nodal metastases limited to the perigastric nodes, or T2N0 tumors. Nodal dissection was done laparoscopically. Conversion to open surgery was permitted when nodal metastases were found along the major branched arteries or serosal invasion by the tumor was suspected during laparoscopic surgery.

LADG was defined as follows: (1) lymph node dissection was performed laparoscopically and (2) mini-laparotomy of $7 \mathrm{~cm}$ was permitted only for reconstruction. Any surgery which did not fulfill the criteria for LADG was defined as open surgery. 
Participants and quality control of surgery

Three hospitals (Kanagawa Cancer Center, Yokohama City University Hospital, and Kanagawa Ashigawa-Kami Hospital) participated in this study. Only three surgeons (T. Yoshikawa, Y. Rino, and Y. Yamamoto), one at each hospital, were responsible for LADG. Each of the three surgeons had experience of more than 300 cases of open gastrectomy, more than 100 cases of laparoscopic cholecystectomy, more than 5 cases of laparoscopic colectomy with lymph node dissection for colon cancer, and more than 5 cases of laparoscopic partial gastrectomy for small submucosal tumors, but none of the three had experience with LADG. All three surgeons had been certified both by the Board of the Japan Surgical Society and by the Japanese Gastroenterological Society. Each surgeon nominated several attending surgeons who could be assistants with LADG. The attending surgeons each had experience of more than 50 cases of open gastrectomy and more than 20 cases of laparoscopic cholecystectomy, but no experience of LADG. These attending surgeons had been certified by the Board of the Japan Surgical Society. They received the following first, second, and third training modules with the above three surgeons, and participated in LADG as laparoscope operators or assistants.

Another two surgeons (T. Fukunaga and M. Kimura), who had LADG experience of more than 50 cases, were invited from other hospitals to act as instructors. The training system was as follows: the first step was to learn how to use the laparoscopic instruments and how to create and show the surgical field; for this training the instructors gave lectures using a videotape that was shown many times. The second step was to use the laparoscopic instruments in a box simulator, in the same way as that shown in the videotape, without the instructors. The third step was to perform a gastrectomy in the animal laboratory in the same way as that shown in the videotape, with lectures and assistance from the instructors. The fourth step was to perform the surgery for gastric cancer patients with assistance from the instructors. The instructors evaluated the trainee's skills and judged whether they could perform LADG surgery without instructors. The fifth step was to perform the surgery without assistance from the instructors. All surgical procedures were recorded on videotape.

An extramural review board, with members having expertise in open surgery for gastric cancer (Dr. M. Ninomiya of the Department of Surgery, Hiroshima City Hiroshima Hospital, Hiroshima, Japan and Dr. N. Hirabayashi of the Department of Surgery, Hiroshima City Asa Hospital, Hiroshima, Japan) checked the videotapes of the operations on the patients. A score sheet was used to evaluate the surgical skills in reconstruction and in dissection by separating each lymph node station. Briefly, the skill was scored as almost perfect $(100 \%)$, good $(80 \%)$, acceptable (60\%), inappropriate (40\%), and unacceptable $(20 \%)$. Mean scores for dissection and reconstruction were calculated separately. If the mean scores were $<60 \%$, such operators were not permitted to do LADG.

\section{Objectives and statistical hypothesis}

The objective of this study was to evaluate the feasibility and safety of LADG. The primary endpoint was surgical morbidity and mortality. Key secondary endpoints were the number of harvested lymph nodes, blood loss, operating time, and overall survival. Surgical morbidity and mortality were evaluated by the National Cancer Institute Common Terminology Criteria for Adverse Events (CTCAE), version 3.0 [11] until 3 months after discharge. Long-term morbidity requiring admission was not evaluated in this study. Data were collected from the first case of surgery with the instructors and were analyzed all together. The safety and feasibility were evaluated in all cases including the training period, because the target was early gastric cancer, which has a high curability rate with open surgery.

The accrual period was set at 5 years. Feasibility and safety were evaluated in the patients enrolled in this period, and the findings were judged by comparing them with the morbidity and mortality of open distal gastrectomy with lymph node dissection (ODG) as a historical control. Termination of the study was prespecified if three treatmentrelated deaths occurred before the initial 60 patients were treated.

This prospective study was approved by the local ethics committees at each institution involved. This report presents the morbidity and mortality (which were the primary endpoints) and other surgical results, including number of harvested lymph nodes, blood loss, and operating time, which were the secondary endpoints in this study.

\section{Results}

No treatment-related death was observed among the initial 60 patients enrolled. Therefore, accrual was continued for 5 years. A total of 193 patients were enrolled in this study between August 2004 and July 2009. The extramural review board checked the videotapes of representative cases twice during this period. The mean scores for dissection and reconstruction were each more than $70 \%$.

The background of the patients is shown in Table 1. Only 18 patients had T2 tumors that required D2 dissection. LADG was initiated for all 193 patients enrolled. The surgical results are summarized in Table 2. D1 + alpha dissection was performed in 7 patients, D1 + beta in 163 , 
Table 1 Patients' background

\begin{tabular}{ll}
\hline Sex $(\mathrm{M} / \mathrm{F})$ & $125 / 68$ \\
Age (years) & $64(24-83)$ \\
$\quad$ Median (range) & 72 \\
Location of tumor & 121 \\
$\quad$ Middle third (M) & \\
Lower third (L) & 13 \\
Tumor progression & \\
$\quad \begin{array}{l}\text { Mucosal N0 tumors (diameter }<1.5 \mathrm{~cm}) \\
\text { with differentiated histology }\end{array}$ & 162 \\
Other T1N0 tumors & 18 \\
T2N0 & 57 (37-99) \\
Body weight (kg) & \\
$\quad$ Median (range) & 22.1 (17.0-34.3) \\
Body mass index & \\
Median (range) &
\end{tabular}

Table 2 Surgical results

\begin{tabular}{ll}
\hline $\begin{array}{l}\text { Maximal wound length }(\mathrm{cm}) \\
\text { Median (range) }\end{array}$ & $6(3.5-18.0)$ \\
Lymph node dissection & 7 \\
D1 + alpha (\#7/\#8a) & 163 \\
D1 + beta (\#7/\#8a/\#9) & 23 \\
D2 & 181 \\
Reconstruction & 12 \\
Billroth-I & \\
Roux-en-Y & $44(9-114)$ \\
No. of harvested lymph nodes & \\
Median (range) & $35(0-2900)$ \\
Blood loss (ml) & $189 / 4$ \\
Median (range) & 2 \\
$<500 />500$ ml & \\
Blood transfusion & $250(135-595)$ \\
Operation time (min) & 6 \\
Median (range) & 4 \\
Conversion to laparotomy & $2(\mathrm{~T} 2 \mathrm{~N} 1$ in 1 and T2N2 in 1) \\
Bleeding & \\
Advanced disease & \\
\hline
\end{tabular}

and D2 in 23. The extent of lymph node dissection defined by the protocol was maintained in all 193 patients. Most patients received Billroth-I reconstruction after distal gastrectomy. The median blood loss was $35 \mathrm{ml}$ and median operation time was $250 \mathrm{~min}$. Conversion to open surgery was seen in 6 patients $(3.1 \%)$; in 4 of these patients conversion was due to bleeding that was not controlled laparoscopically and in 2 conversion was due to advanced disease (T2N1 in 1 patient and T2N2 in the other).
Table 3 Pathological findings

\begin{tabular}{ll}
\hline Pathological type & \\
Differentiated/undifferentiated & $77 / 116$ \\
$\mathrm{~T}$ & \\
$\mathrm{~T} 1(\mathrm{~m} / \mathrm{sm})$ & $103 / 71$ \\
$\mathrm{~T} 2(\mathrm{mp} / \mathrm{ss})$ & $12 / 6$ \\
$\mathrm{~T} 3(\mathrm{se})$ & 1 \\
$\mathrm{~N}$ & \\
$\mathrm{~N} 0$ & 162 \\
$\mathrm{~N} 1$ (perigastric nodes) & 23 \\
$\mathrm{~N} 2$ (along major branched arteries) & 8 \\
$\mathrm{M}$ & \\
$\mathrm{M} 0$ & 192 \\
M1 (positive peritoneal cytology) & 1 \\
\hline$m / s m$ mucosal/submucosal, $\mathrm{mp} / \mathrm{ss}$ muscularis propria/subserosal, se \\
serosal
\end{tabular}

Table 4 Morbidity and mortality

\begin{tabular}{ll}
\hline Any complication $(\geq$ grade 2$)$ & $3(1.6 \%)$ \\
Anastomotic leakage & $1(0.5 \%)$ \\
$\quad$ Grade 2 & $1(0.5 \%)$ \\
Pancreatic fistula & \\
$\quad$ Grade 2 & $1(0.5 \%)$ \\
Mechanical obstruction & \\
$\quad$ Grade 3 & 0 \\
Other complications & 1 \\
$\quad \geq$ Grade 2 & 0 \\
Re-operation before discharge & \\
In-hospital mortality & $9(7-37)$ \\
Duration of hospitalization after surgery (days) \\
Median (range)
\end{tabular}

The pathological findings are shown in Table 3 . One patient had advanced tumors invading the serosa, as well as peritoneal cytology. Nodal metastases along the major branched arteries were observed in 8 patients.

The morbidity and mortality data are shown in Table 4. Grade 3 or more morbidity was observed in 1 patient, who had a mechanical obstruction that required surgery. There was no re-operation and no mortality in the 3 months after discharge.

The number of cases required until the trainee surgeons could act as LADG surgeons without a trainer was 3 for each of the three surgeons. When comparing the data between the training period $(n=9)$ and the period when the operators acted without a trainer $(n=174)$, the median operation time was significantly longer in the former period (355 min) than in the latter $(247.5 \mathrm{~min})(p=0.015)$. The median blood loss was greater in the former $(150 \mathrm{ml})$ than 
in the latter period $(32.5 \mathrm{ml})$, but the difference did not reach statistical significance $(p=0.084)$. During the training period, no patient developed any complications of $\geq$ grade 2 .

\section{Discussion}

In the present study, the median blood loss was only $35 \mathrm{ml}$ and median operation time was approximately $4 \mathrm{~h}$. Blood loss in ODG is reported to range from 55 to $488 \mathrm{ml}$, which is slightly more than the present result [12]. The operation time for ODG ranges from 124 to $228 \mathrm{~min}$ [12]. Thus, LADG in this study took slightly more time than the reported times for ODG, but the difference was marginal. On the other hand, no mortality was observed in this study, and morbidity was $1.6 \%$. Moreover, the incidence of pancreatic fistula or anastomotic leakage was only $0.5 \%$. The rates of anastomotic leakage and pancreatic fistula following ODG are reportedly 0.6-2.7 and 0.6\%, respectively [13-15]. We note that Katai et al. [8] first reported the feasibility and safety of LADG in a large-scale prospective phase II trial in Japan. They demonstrated that the median blood loss was $43 \mathrm{ml}$ and median operation time was $250 \mathrm{~min}$. The incidences of pancreatic fistula and anastomotic leakage were $1.7 \%$ each. The present prospective study showed surgical results that were similar to those in the report by Katai et al. [8]. Thus, the present results suggest that LADG could be initiated and performed feasibly and safely if surgeons with much experience of open gastrectomy and laparoscopic surgery receive adequate training for LADG.

In several case series reported during the initial introduction of LADG, blood loss was reportedly over $200 \mathrm{ml}$ and surgical morbidity was around $10 \%$ [12]. However, surgical results have since improved [8]. LADG has been refined over time. One possible reason is the easy access to surgical skills achieved by using videotapes of procedures, and these can be easily taken during laparoscopic surgery. In addition, technical skills are freely shared during medical and intramural conferences. Improved techniques can be easily spread, and the development of tools has improved techniques. Innovations in cutting tools include an ultrasonic cutting and coagulating system and a bipolar vessel sealing system, which may help to reduce bleeding and shorten the operation time.

The training period in the present study was very short. Only 3 cases were required for each of the three trainee surgeons to be able to be accredited as a LADG surgeon. Although the operating time was longer in the training period than that in the period when the operators no longer required training, no surgical morbidity was observed in the training period. To avoid surgical morbidity such as pancreatic leakage, it is essential to cut along the appropriate line and to separate the correct layer even though it takes a long time. As the three surgeons had much experience with open gastric surgery, they understood the appropriate line along which to cut and the correct layer to separate. Thus, the appropriate surgery could be more important than the operation time when initiating LADG. However, blood loss was slightly high in the training period, which may have been due to the surgeons' immature skills in laparoscopic operation or in stopping the bleeding. As reconstruction was done by an extracorporeal approach in this study, anastomosis-related morbidity was few even in the training period.

According to previous studies, LADG was regarded as a very complicated procedure and surgeons required 50-90 cases to obtain sufficient skills [16-18]. Recently, Mochizuki et al. [19] reported that the minimal number of cases required to obtain sufficient skills was 25 if surgeons had strict surgical backgrounds and were well trained. In the present study, the minimal number of cases was only 3 . Practical training in the present study was less than that reported by Mochizuki et al. [19]. Their operators did not receive lessons by videotape. Surgical technique can depend on the surgical field, the motion of the instruments, and the method itself. Simulation of surgical technique is possible by videotape without the operator doing practical training. Moreover, the backgrounds of the surgeons in their study were different from those in our study. In their study, the surgeons' only experience of laparoscopic surgery was with cholecystectomy, while the surgeons in the present study had laparoscopic experience with cholecystectomy, partial gastrectomy, and colectomy with nodal dissection for colon cancer. Our results suggested that only several cases were required for surgeons to obtain the skills necessary for LADG if they had sufficient knowledge of gastric cancer surgery, sufficient skill with laparoscopic surgery, and adequate training.

Generally, surgeons begin with an easy operation and go forward to the next step. Experience with laparoscopic surgery would be similar. Circumstances around surgery in general hospitals have changed dramatically from the time when the technique of LADG was first developed. The technique of LADG is now established and sophisticated. The instruments have been improved. Basic laparoscopic surgery is performed widely in Japan. The employment of laparoscopic colectomy is also spreading. Before initiating LADG in general hospitals, surgeons may now have experience of laparoscopic cholecystectomy, laparoscopic partial gastrectomy, and laparoscopic colectomy. Thus, in the present study, the backgrounds of the surgeons may be much closer to those in current circumstances in general hospitals compared with the backgrounds of the surgeons in Mochizuki's report [19]. Although our study achieved 
excellent results, it is still unclear whether our methods are applicable to other surgeons with similar surgical backgrounds. A confirmatory study is necessary.

There are several reasons for the excellent results of the present study: first, the surgeons had much experience of laparoscopic surgery. They were used to laparoscopic surgery. Second, the surgeons received adequate stepby-step training. Being able to view a training videotape many times is extremely important for learning about the surgical field and work of the instrument. Only after obtaining the image of LADG were surgeons trained to do the same work in the surgery. Third, surgeons who had much experience of LADG acted as instructors. It usually requires an extended period to improve and refine surgical skills by practice alone. The instruction by expert surgeons standardized the technique [20,21], and LADG could be performed correctly for the first patient. All the procedures were checked by the instructors. Fourth, all the responsible surgeons had much experience of open gastrectomy. An understanding of the anatomy and dissection for gastric cancer may help surgeons perform LADG. Moreover, reconstruction was done by an extracorporeal approach following the method developed and standardized in open surgery. All of the surgeons had experience with this reconstruction method. Fifth, the surgical skills were checked by an extramural review board. The surgeons were careful to avoid criticism from the review.

Special attention is required in the interpretation of the present results. There were several limitations of this study. First, there was a possibility of bias in the selection of the patients. This study was a prospective one, performed to evaluate the feasibility and safety of LADG. Only selected patients who satisfied the entry criteria were enrolled in the trial. Moreover, the technique was reviewed by extramural board members. However, some surgeons may have hesitated to operate on elderly or obese patients, or those who had severe co-morbidities even though they satisfied the entry criteria. Therefore, it is unclear whether the present results are applicable to such patients. Second, the trainees in this study had much experience of open gastrectomy and laparoscopic surgery. These methods and results may not be applicable to less experienced surgeons.

In conclusion, LADG could be initiated and performed feasibly and safely if surgeons with much experience of open gastrectomy and laparoscopic surgery received adequate training.

Acknowledgments This study was supported in part by the nonprofit organization Kanagawa Standard Anti-cancer Therapy Support System (KSATTS). We greatly appreciate Dr. Motoki Ninomiya of the Department of Surgery, Hiroshima City Hiroshima Hospital, Hiroshima, Japan and Dr. Naoki Hirabayashi of the Department of Surgery, Hiroshima City Asa Hospital, Hiroshima, Japan for their great contribution to the quality control of this study.

\section{References}

1. Kitano S, Iso Y, Moriyama M, Sugimachi K. Laparoscopy-assisted Billroth I gastrectomy. Surg Laparosc Endosc 1994;4(2):146-8.

2. Fujiwara M, Kodera Y, Kasai Y, Kanyama Y, Hibi K, Ito K, et al. Laparoscopy-assisted distal gastrectomy with systemic lymph node dissection for early gastric carcinoma: a review of 43 cases. J Am Coll Surg. 2003;196:75-81.

3. Sakuramoto S, Kikuchi S, Kuroyama S, Futawatari N, Katada N, Kobayashi N, et al. Laparoscopy-assisted distal gastrectomy for early gastric cancer: experience with 111 consecutive patients. Surg Endosc. 2006;20:55-60.

4. Kitano S, Shiraishi N, Fujii K, Yasuda K, Inomata M, Adachi Y. A randomized controlled trial comparing open vs laparoscopyassisted distal gastrectomy for the treatment of early gastric cancer: an interim report. Surgery. 2002;131:S306-11.

5. Kim MC, Kim KH, Kim HH, Jung GJ. Comparison of laparoscopy-assisted by conventional open distal gastrectomy and extraperigastric lymph node dissection in early gastric cancer. J Surg Oncol. 2005;91:90-4.

6. Kim YW, Baik YH, Yun YH, Nam BH, Kim DH, Choi IJ, et al. Improved quality of life outcomes after laparoscopy-assisted distal gastrectomy for early gastric cancer: results of a prospective randomized clinical trial. Ann Surg. 2008;248:721-7.

7. Kitano S, Shiraishi N, Uyama I, Sugihara K, Tanigawa N. A multicenter study on oncologic outcome of laparoscopic gastrectomy for early cancer in Japan. Ann Surg. 2007;245:68-72.

8. Katai H, Sasako M, Fukuda H, Nakamura K, Hiki N, Saka M, et al. Safety and feasibility of laparoscopy-assisted distal gastrectomy with suprapancreatic nodal dissection for clinical stage I gastric cancer: a multicenter phase II trial (JCOG 0703). Gastric Cancer. 2010;13:238-44.

9. Japanese Gastric Cancer Association. Japanese classification of gastric carcinoma, 2nd English edition. Gastric Cancer 1998;1: $10-24$.

10. Nakajima T. Gastric cancer treatment guidelines in Japan. Gastric Cancer. 2002;5:1-5.

11. Trotti A, Colevas AD, Setser A, Rusch V, Jaques D, Budach V, et al. CTCAE v3.0: development of a comprehensive grading system for the adverse effects of cancer treatment. Semin Radiat Oncol. 2003;13:176-81.

12. Shiraishi N, Yasuda K, Kitano S. Laparoscopic gastrectomy with lymph node dissection for gastric cancer. Gastric Cancer. 2006;9:167-76.

13. Naka T, Ishikura T, Shibata S, Yamaguchi Y, Ishiguro M, Yurugi E, et al. Laparoscopy-assisted and open distal gastrectomies for early gastric cancer at a general hospital in Japan. Hepatogastroenterology. 2005;52:293-7.

14. Sasako M, Katai H, Sano T, Maruyama K. Management of complications after gastrectomy with extended lymphadenectomy. Surg Oncol 2000;9(1):31-4.

15. Dulucq JL, Wintringer P, Stabilini C, Solinas L, Perissat J, Mahajna A. Laparoscopic and open gastric resections for malignant lesions: a prospective comparative study. Surg Endosc. 2005;19:933-8.

16. Kim MC, Jung GJ, Kim HH. Learning curve of laparoscopyassisted distal gastrectomy with systemic lymphadenectomy for early gastric cancer. World J Gastroenterol. 2005;11:7508-11.

17. Kunisaki C, Makino H, Yamamoto N, Sato T, Oshima T, Nagano $\mathrm{Y}$, et al. Learning curve for laparoscopy-assisted distal gastrectomy with regional lymph node dissection for early gastric cancer. Surg Laparosc Endosc Percutan Tech. 2008;18:236-41.

18. Zhang X, Tanigawa N. Learning curve of laparoscopic surgery for gastric cancer, a laparoscopic distal gastrectomy-based analysis. Surg Endosc. 2009;23:1259-64. 
19. Mochizuki Y, Kodera Y, Fujiwara M, Ito S, Misawa K, Kanemitsu K, Ito S. Single-institute prospective trial of laparoscopyassisted distal gastrectomy with systemic lymph node dissection for early gastric carcinoma. Gastric Cancer. 2011.

20. Fukunaga T, Hiki N, Tokunaga M, Nohara K, Akashi Y, Katayama $\mathrm{H}$, et al. Left-sided approach for suprapancreatic lymph node dissection in laparoscopy-assisted distal gastrectomy without duodenal transection. Gastric Cancer. 2009;12:106-12.

21. Hiki N, Fukunaga T, Yamaguchi T, Nunobe S, Tokunaga M, Ohyama S, et al. The benefits of standardizing the operative procedure for the assistant in laparoscopy-assisted gastrectomy for gastric cancer. Langenbecks Arch Surg. 2008;393:963-71. 